\title{
Erratum to: Western Pine Beetle Populations in Arizona and California Differ in the Composition of Their Aggregation Pheromones
}

\author{
Deepa S. Pureswaran ${ }^{1} \cdot$ Richard W. Hofstetter ${ }^{2} \cdot$ Brian T. Sullivan $^{3}$. \\ Amanda M. Grady ${ }^{4}$ Cavell Brownie ${ }^{5}$
}

Published online: 7 June 2016

(C) Springer Science+Business Media New York 2016

\section{Erratum to: J Chem Ecol (2016) \\ DOI 10.1007/s10886-016-0696-9}

The five Dendroctonus frontalis that were sampled in Pureswaran et al. (2016) and included in the histogram analysis of Fig. 3 were males, not females. Thus in the the Y-axis title for Fig. 3, "females" should instead be "beetles". The corrected Fig. 3 with legend is below.

Additionally, there is an incorrect statement regarding beetle sex in the METHODS:

"MANOVA. The ratios of endo/exo-production by individual females were plotted in a histogram for $D$. brevicomis samples in addition to pheromone production data for five Arizona-derived female $D$. frontalis reported by Pureswaran et al. (2016). For these five beetles the pheromone sampling techniques were essentially identical to those used in the present paper."

The online version of the original article can be found at http://dx.doi.org/ 10.1007/s10886-016-0696-9

Deepa S. Pureswaran

deepa.pureswaran@canada.ca

1 Canadian Forest Service, Laurentian Forestry Centre, 1055, rue du PEPS, Quebec City, QC G1V 4C7, Canada

2 School of Forestry, Northern Arizona University, Box 15018, Flagstaff, AZ 86011, USA

3 USDA Forest Service, Southern Research Station, Pineville, LA 71360, USA

4 USDA Forest Service, Forest Health Protection, AZ Zone, Flagstaff, AZ 86001, USA

5 Department of Statistics, North Carolina State University, Raleigh, NC 27695, USA
The corrected portion of the above should read "...data for five Arizona-derived male $D$. frontalis reported by Pureswaran et al. (2016)."

There also was an incorrect statement in the RESULTS section:

"The endo/exo-brevicomin ratios for five sampled D. frontalis females from Arizona (Pureswaran et al. 2016) ranged from 8.00 to 26.5 , and grouped with the lower half of the Arizona cluster."

The corrected portion should read "The endo/exo-brevicomin ratios for five sampled $D$. frontalis males from Arizona...".

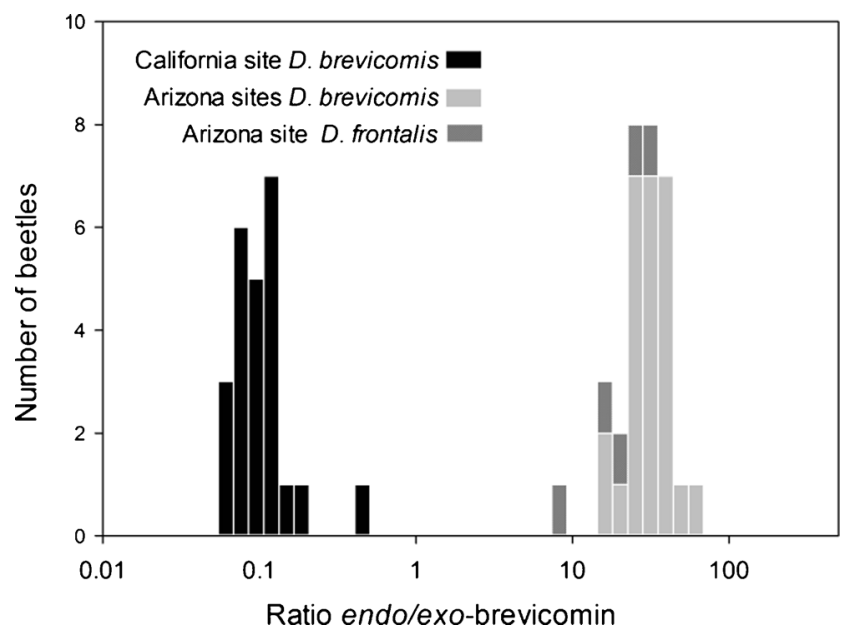

Fig. 3 Histogram illustrating the distribution of raw ratios of endo/exobrevicomin in volatiles isolated from paired female Dendroctonus brevicomis from a site in northern California and two sites in northern Arizona (both pooled). Data for five male Dendroctonus frontalis Zimmermann collected in northern Arizona and sampled by identical methods as D. brevicomis are also shown 